\title{
Research Resources
}

\author{
A summary of new products and services for materials research...
}

\begin{abstract}
Medium Energy Ion Scattering Spectroscopy (MEIS) System: Ultrahigh vacuum system for the structural analysis of solid surfaces and interfaces with medium energy ion scattering $\left(50-330 \mathrm{keV} \mathrm{H}^{+}\right.$or $\mathrm{He}^{+}$) includes a main chamber with a sample manipulator and a specially designed toroidal electrostatic analyzer. The chamber allows combining MEIS with other surface analysis techniques such as low energy electron diffraction and Auger electron spectroscopy in situ. Designed to detect backscattered ions over an angle of $30^{\circ}$ simultaneously, the toroidal analyzer has an energy resolution of $4 \times 10^{-3}$. Ion beam dose and surface contamination are reduced considerably, and the resulting depth resolution is $3-5 \AA$. The combination of MEIS with the effects of channeling and blocking allows the high precision determination (up to $0.02 \AA$ ) of the geometric arrangements of atoms in a surface. The special sample loading chamber makes it possible to exchange samples within minutes without breaking the ultrahigh vacuum of the main chamber $\left(10^{-10} \mathrm{mbar}\right.$
\end{abstract}

range). High Voltage Engineering Europa B.V., Amsterdamseweg 63, 3812 RR Amersfoot, P.O. Box 99, 3800 AB Amersfoort, The Netherlands; 31-33-19741.

\section{SIMS Instrument for Advanced Semicon-} ductor Analysis: The IX70S is based on a powerful magnetic sector mass spectrometer capable of mass resolution in excess of $\mathrm{M} /{ }_{\Delta \mathrm{M}}=40,000$. A choice of primary ion sources provides optimum performance in various analysis modes. Features include: ultrahigh vacuum construction to provide improved detection limits; high intensity, mass filtered oxygen and cesium ion sources for concentration - depth profiling; low energy fast atom bombardment for optimized depth resolution; microfocussed liquid metal ion beams for $1000 \AA$ imaging of device structures; and fast framestore computer system for three-dimensional data storage and image processing. VG Ionex Limited, Charles Avenue, Maltings Park, Burgess Hill, West Sussex RH15 9TQ, U.K.; 44-4446-46333.
Analytical X-Ray Systems: Brochure describes instrumentation including: SRS 300 sequential $x$-ray spectrometer capable of analyzing boron to uranium by changing parameters; MRS $400 \mathrm{MP}$ simultaneous $x$ ray spectrometer for applications demanding rapid analysis/high sample throughput (more than 100 samples in 8-hours); D 500 $x$-ray diffractometer; DACO-MP, a microprocessor-based theta-theta version of the D 500; software for $x$-ray spectroscopy, $x$-ray diffraction, and laboratory data management; and generators. Siemens AG, E 68 A, Analytical Systems, P.O. Box 21 1262, D 7500 Karlsruhe 21, West Germany; 49-721-595-4295.

\section{UHV Compatible Elemental Standards:} Ultrahigh vacuum compatible elements contain no synthetic embedding media, are individually polished, and are user removable for replacement or customization. Thirty-six elements, each about $\frac{1}{8}$ inch in diameter, and a Faraday cup are provided in a 1-in.-diameter SS304 holder. The

\section{Pressure Measurementand Control}

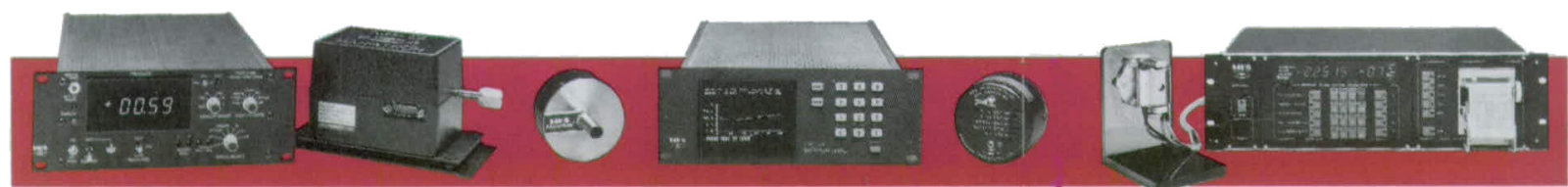

Type 270B and Type 390/398 High Accuracy Baratron $^{\oplus}$ System Temperature controlled at $45^{\circ} \mathrm{C}$ for superior zero and span stability. Accuracy $0.08 \%$ of Reading, optional $0.05 \%$.
Type 122/127/112A - Pressure Programmer/ Display and 100 Series Baratron Sensors Computer based instrument, with application specific software to measure, display, analyze and contról process parameters for pressure, vacuum, and flow.
Spinning Rotor Friction Gauge The transfer standand for vacuum gauge calibration in the range of $10^{-7}$ to $10^{-2}$ torr with accuracy of $1 \%$ of Reading $\pm 3 \times 10^{-8}$ torr.

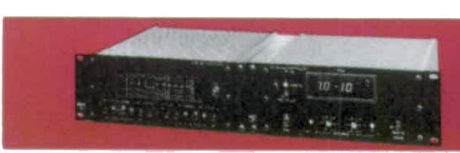

Type 290 Ion Gauge Controllers High vacuum pressure measurement system with Charge-Rate* circuitry to allow continuous ranging from $10^{-3}$ to $10^{-9}$ torr.

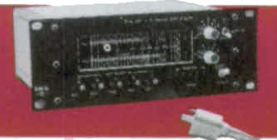

Type 286/287 Thermocouple Vacuum Gauges

Economical vacuum process pressure measurements from 2 to $10^{-2}$ torr in non-corrosive environments. 2 channel and 5 channel.
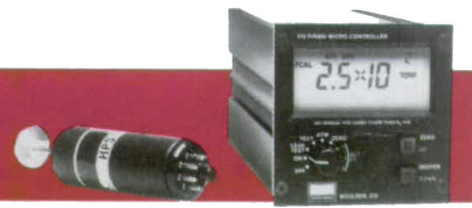

HPS Series 315 Pirani Gauge System Continuous high performance general purpose vacuum measurement from $10^{-3}$ to 100 torr with discrete points to atmosphere. Gauge tube features temperature compensation for improved Pirani Gauge stability.
Our complete line of pressure measurement and control instruments represent the ultimate in electronic manometer simplicity, economy and performance.
'US. Patent No. 4,0355,720 Foreign Patents and Patents Pending

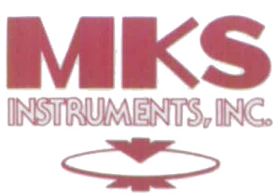

Six Shattuck Road Andover, MA 01810 Tel. (617) 975.2350 Telex: (617) 975-0094 Fax: (617) 975-0093

Please visit Booth No. 306 at the MRS Show. 
holder, micro-engraved for ease in locating the desired position, is suitable for use with Auger electron spectrometers, SEMs with $x$-ray detectors, electron probe microanalyzers, small spot ESCA instruments, and other macro- or microanalytical devices. Geller Micro Ånalytical Laboratory, One Intercontinental Way, Peabody, MA; (617) 535-5595.

\section{Microprocessor-Controlled Sputtering} System: Fully automated Model XM-90 with expanded flexibility and process recipe control can regulate parameters such as load-lock pressure, deposition time and power, and substrate heater temperatures. Process recipe control for each of the four in-line process stations, along with the ability to deposit three separate times at a single process station, allows the user to interlayer sputtered films reactively and nonreactively. The XM-90 processes gallium arsenide and lapped silicon, and deposits films of titanium tungsten, gold, copper, or chromium for "bump" metalization in tape-automated bonding (TAB). For deposition of precious metals, the XM-90 incorporates plasma confinement shielding to facilitate the recovery of sputtered material. Varian Associates, Semiconductor Equipment Group, 611 Hansen Way, Palo Alto, CA 94303; (800) 544-4636.
Uitrasonic Nondestructive Characterization: Thirty-page technical bulletin offers a comprehensive explanation of Ultran's transducers for ultrasonic nondestructive testing, evaluation, and characterization. Five basic acoustic series are available in a frequency range of $<100 \mathrm{kHz}$ to $>100$ $\mathrm{MHZ}$ and in a dimensional range from $<0.75$ to $>125 \mathrm{~mm}$. Transducer designs feature standard and VHF immersion, standard contact, anglebeam/shearwave, transmitter/receiver, replaceable membrane, delay line, high temperature, dry coupling, air/gas propagation, $0^{\circ}$ incident shearwave, lambda, radiused element transducers, and more, including customdesigned transducers. Additional brochures describe the company's services in materials testing and characterization, feasibility analysis, R\&D, and consulting. Ultran Laboratories, 139R North Gill Street, State College, PA 16801; (814) 2389083.

Cadmium Plating Package: Five-part compilation of booklets and articles on cadmium's electroplating capabilities, design considerations, safe handling, and supply sources is a single-source reference for design engineers, industrial specifiers, and other materials decision makers. Included are: profiles of job shops that plate cad- mium; a 16-page booklet on the functional benefits of cadmium-plated components; a directory of plating facilities (names, addresses, and phone numbers of cadmium electroplaters in the U.S. and Canada); and a technical paper that discusses cadmium's role in cars. Cadmium Council, 292 Madison Avenue, New York, NY 10017; (212) 578-4750.

Ion Beam Interactions Software: The VG SUSPRE package for the fast calculation of ion beam interactions with solids can calculate ion implantation range profiles for all implant species and substrates and is valid for ion energies up to a few hundred $\mathrm{keV}$. It can also predict the ion-induced damage profile and electronic and nuclear energy loss deposition. In calculating ion beamsurface interactions, the program predicts sputtering yields, erosion rates, and ion beam mixing effects. Applications include depth profiling in surface analysis, ion beam etching, and ion-assisted growth techniques. Available for IBM-PC and DEC PDP-11. VG Ionex Limited, The Maltings, Burgess Hill, West Sussex RH15 9TQ, U.K.; 44-4446-46333.

\section{Flow Measurement and Control}

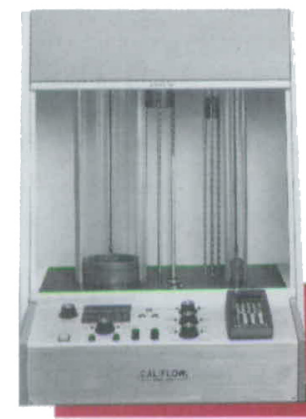

CAIIFLOW High Accuracy

Flow Rate Calibration Standard

The primary standard for gas flow calibration from $20 \mathrm{sccm}$ to $50 \mathrm{sim}$ with accuracy of $0.2 \%$ of Reading.

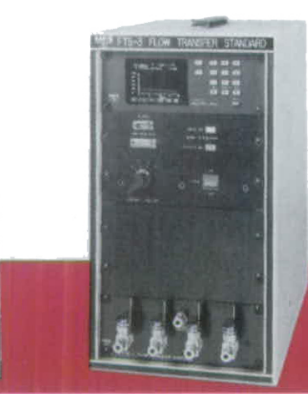

Type FTS - Flow Transfer Standard System Conveniently packaged system employing MKS 1259 high performance flow controllers, 112A Flow Programmer/ Display; manifolding and uninterruptable power supply for ease of portability.

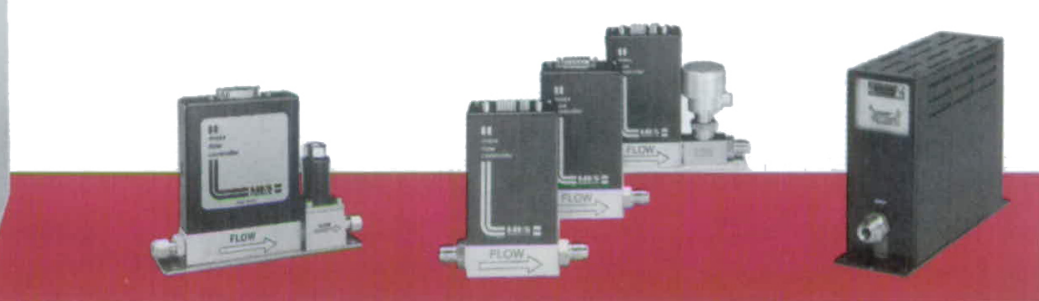

Type 1259/2259 High Performance Thermal Mass Flowmeter/Controller Transfer standard performance in a mass flow controller. Uses MKS Patented* flow sensing technique. $0.8 \%$ F.S. accuracy.
Type 1159 and 1160 Thermal Mass Flow Controllers

High performance features include patented MKS flow sensing technique, low pressure drop fast response time, and long term therma stability.
Type 1150 Vapor Source Flow Controller All metal, high temperature pressure measurement based instrument for direct measure ment and control of low vapor pressure sources, vaporized liquid sources and sublimed solid sources.
MKS has manufactured quality instruments for the accurate measurement and control of pressure and flow for more than 25 years. Let us help you find the best measuring or controlling technique for your application. Call us at 1-800-227-8766.
"US. Patent No. 4,464,932 Foreign Patents and Patents Pending

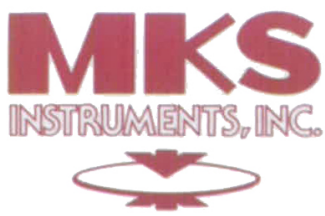

Six Shattuck Road Andover, MA 01810 Tel (617) 975.2350 Telex: (617) 975-0094 Fax: (617) 975.0093

Please visit Booth No. 306 at the MRS Show. 


\section{NEW INSTRUMENTS FOR TESTING THE MECHANICAL PROPERTIES OF THIN FILMS AND COATINGS}

\section{REVETEST SCRATCH TESTER}

The CSEM Revetest is a new instrument for measuring the mechanical strength (adhesion and intrinsic cohesion) of hard and brittle coatings produced by CVD (chemical vapor deposition) and PVD (physical vapor deposition) techniques.

- Easy to use

- Automatic operation

- Quantitative results

- Frictional coefficient option

- Microscope option

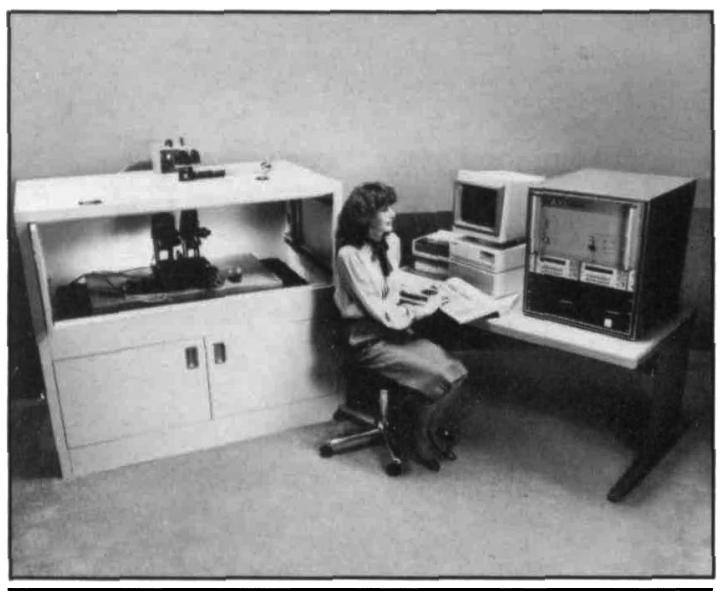

\section{TRIBOMETER PIN-ON-DISC MACHINE}

The CSEM Tribometer is a pin-on-disc type instrument for the precise measurement of the friction and wear properties of materials and the functional lifetime of tribological coatings.

- Easy to use

- Complete with discs and ball holders

See our display at the AVS National Convention in Anaheim, California, Booths 601-607 and the MRS Fall Show in Boston, Massachusetts, Booths 802 and 803.

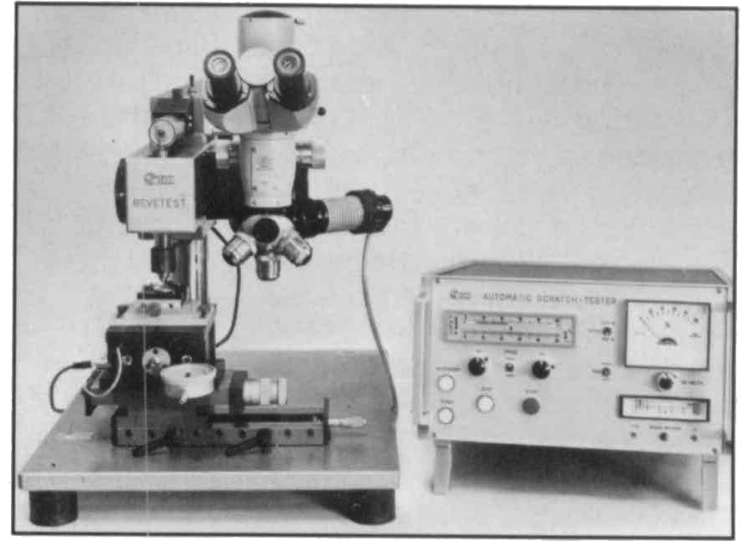

\section{NANO INDENTER}

The NANO Indenter is a new instrument for measuring the mechanical properties of a surface on a submicron scale. The instrument measures the hardness and elastic modulus of a material surface.

- No imaging required

- Complete computer acquisition of penetration depth and load

- Differentiates between elastic and plastic strains

- Data from indentations as shallow as $20 \mathrm{~nm}$

- Programmable, indent patterns, approach rates, loading and unloading

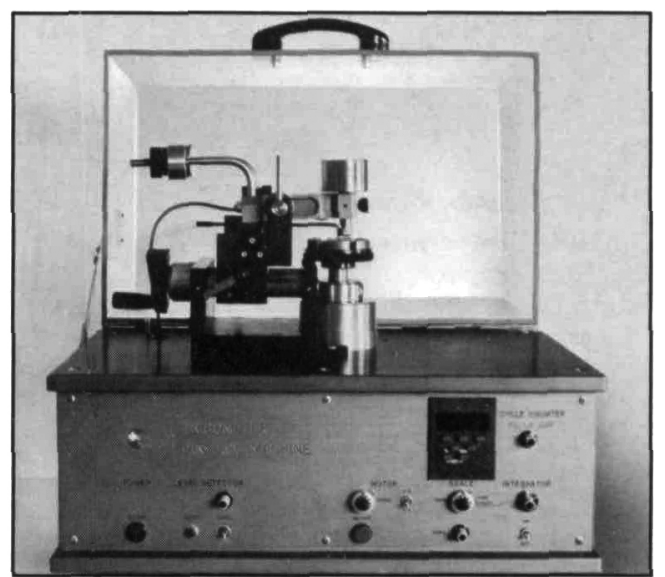




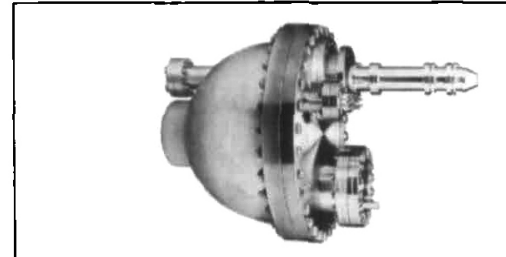

VSW Hemispherical Analyzers

- High resolution optics and electronics

- $50 \mathrm{~mm}, 100 \mathrm{~mm}$ and $150 \mathrm{~mm}$ radius

- Externally variable slit

- Flange and goniometer mounting

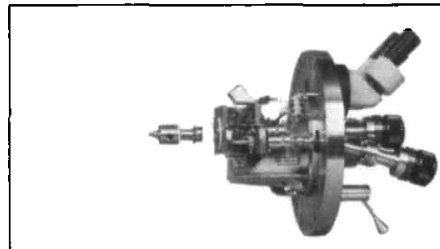

\section{Scanning Tunnelling Microscope}

- Atomic resolution

- UHV mounting with sample transfer

- I-V spectroscopy

- Complete electronics and imaging software

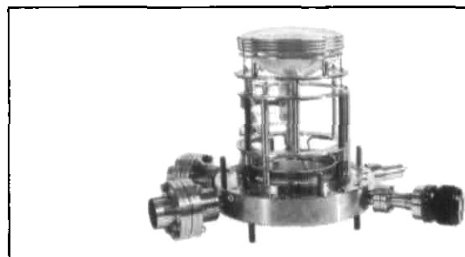

\section{Retractable Rear View LEED}

- Up to 4 grids

- $50 \mathrm{~mm} \mathrm{Z}$ motion

- Custom length and 8" or 6" optics

- Miniature electron gun

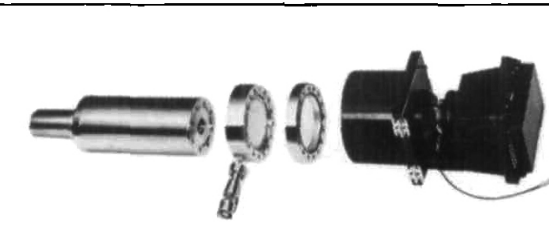

\section{RHEED}

- 10-30 kV self bias electron gun and power supply

- Hand-held remote control

- Complete package, screen, viewport, shutter and camera options

- High stability and reproducibility

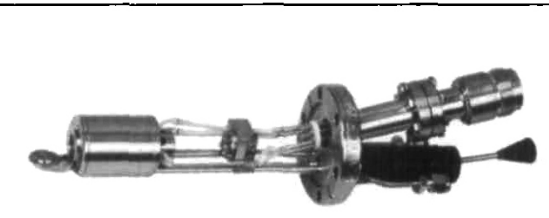

\section{Knudsen Cells}

- 2.75" conflat and cluster mounting

- Boron nitride or graphite crucible

- Integral $\mathrm{H}_{2} \mathrm{O}$ or $\mathrm{LN}_{2}$ cooling

- Digital temperature control

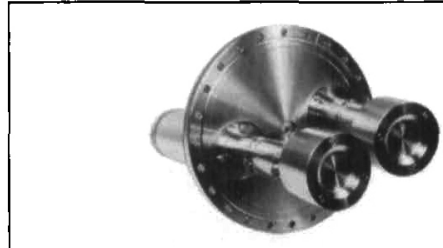

\section{Magnetrons}

- $2^{\prime \prime}, 3^{\prime \prime}$ and $\left(6^{\prime \prime}\right.$ rectangular) targets

- RF \& DC operation

- UHV versions on conflat flange

- Mount in any orientation

\section{- Miciosclica We deliver innovation.}

Microscience Inc., Headquarters: 41 Accord Park Drive, Norwell MA 02061 (617) 871-0308

Sales/Service Offices: San Jose CA (408) 943-0111; Allentown PA (215) 398-1501; Lewisville TX (214) 221-9483

See our display at the AVS Show, Booths 601-607, Nov 3-6 1987. 